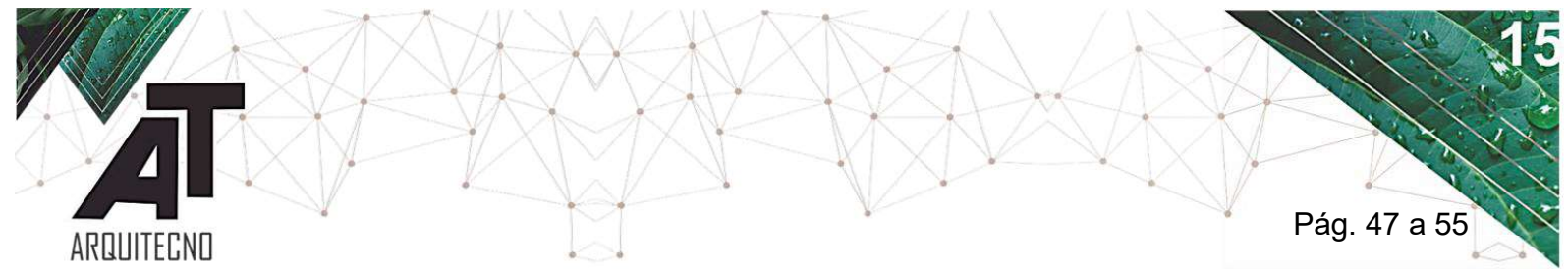

\title{
Técnica, tectónica y tecnología en el proyecto de la vivienda contemporánea: Una experiencia pedagógica desde la práctica docente de grado
}

\section{Contemporary housing planning tecnique, tectonique and tecnology: A pedagogical experience from grade teaching practice}

Diego Martín Fiscarelli

Laboratorio de Tecnología y Gestión Habitacional LATEC. FAU. UNLP, Argentina.

\section{RESUMEN}

dfiscarelli@fau.unlp.edu.ar.

\begin{abstract}
Este trabajo presenta una discusión teórica a partir de los resultados de las jornadas de trabajo en taller del WorkShop "Técnica, tectónica y tecnología para la vivienda contemporánea: estrategias y recursos proyectuales", desarrollado en la Facultad de Arte, Diseño y Urbanismo de la Universidad Nacional de Asunción del Paraguay. Interpelando la vivienda contemporánea a partir de su materialidad, y relativizando su concepción como objeto en permanente transformación, se propuso construir un espacio de reflexión colectiva y crítica arquitectónica. En lo específico, los encuentros intensivos de trabajo alentaron la construcción conceptual de dispositivos tecnológicos, organizativos y morfológicodimensionales, con el objeto de habilitar soluciones alternativas para definir los rasgos esenciales de la vivienda de nuestro tiempo. Se revisaron estrategias y recursos proyectuales, que haciendo foco en la tecnología, permitieran resolver como casos instrumentales, una serie de organizaciones familiares diversas.
\end{abstract}

Como parte de la discusión, se exponen aquellos casos que contribuyeron a definir una tectónica para la vivienda contemporánea, en oposición al carácter menos permeable a las transformaciones, aspecto heredado de la concepción vitruviana y representado por el firmitas.

\section{ABSTRACT}

This work presents a theoretical discussion based on the results of the work sessions in the WorkShop "Técnica, tectónica y tecnología para la vivienda contemporánea: estrategias y recursos proyectuales", developed at the Faculty of Art, Design and Urbanism of the National University of Asunción del Paraguay. Questioning contemporary housing based on its materiality, and relativizing its conception as an object in permanent transformation, it was proposed to build a space for collective reflection and architectural criticism. Specifically, intensive work meetings encouraged the conceptual construction of technological, organizational, and morphological-dimensional devices, with the aim of enabling alternative solutions to define the essential features of housing in our time. Project strategies and resources were reviewed, which, focusing on technology, allowed solving, as instrumental cases, a series of diverse family organizations.

As part of the discussion, those cases that contributed to defining a tectonics for contemporary housing are exposed, as opposed to the less permeable character to the transformations, an aspect inherited from the Vitruvian conception and represented by firmitas.

PALABRAS CLAVES: enseñanza, taller, programa complejo.

KEY WORDS: teaching, workshop, Complex Program.

FECHA DE RECEPCIÓN: 08/03/2020 | FECHA DE ACEPTACIÓN: 10/05/2020

DOI: http://dx.doi.org/10.30972/arq.0154387 
Técnica, tectónica y tecnología en el proyecto de la vivienda contemporánea: Una experiencia pedagógica desde la práctica docente de grado

\section{INTRODUCCIÓN}

A pesar de que las sociedades latinoamericanas exponen gradualmente muestras de cambio en los modos de habitar (Sarquis, 2005) que incluyen -entre otras- conformaciones familiares no tradicionales y nuevas modalidades de vínculo y relaciones laborales podemos afirmar que existe una distancia considerable entre el estado del debate de estas cuestiones en términos sociológicos, y la consideración de mecanismos proyectuales que propongan alternativas viables. En otros términos, a diferencia de lo que ocurre con las Unidades Domésticas, cuyo Ciclo Vital Familiar (Torrado, 2003) implica desplazamientos, cambios de tamaño, de estructura y de necesidades en fases relativamente breves y dinámicas, la vivienda urbana en la actualidad aún se concibe como una porción de espacio privado destinado al alojamiento, con limitadas posibilidades tecnológico-constructivas que faciliten adecuaciones físicas y/o de uso. En este sentido, se reconoce que la persistente conceptualización, diseño y materialización de la vivienda como programa como un constructo arquitectónico rígido, definitivo y tipificado, cuyos ámbitos se conciben a partir de actividades únicas y específicas constituye un evidente equívoco frente a la diversidad y variabilidad de requerimientos que surgen durante el lapso de su vida útil.

Comprendemos entonces que ante esta aparente contradicción entre vivienda estática -condicionada en su capacidad de transformación- y demandas humanas variables alienta la necesidad de producir, desde los aspectos tecnológico-constructivos del proyecto un margen de compatibilidad entre ambos polos de esta tensión. Incluso desde el proyecto, urbano la rigidez y permanencia propias de la concepción tradicional de la ciudad y de sus parámetros asociados -control, crecimiento, estabilidadhan cedido ante la indeterminación y mutabilidad de las ciudades contemporáneas, permeables en mayor medida a incorporar mecanismos abiertos con capacidad de evolución, trasformación y adaptación.

Atendiendo a estas cuestiones, presentamos la noción de adaptabilidad como la capacidad de una edificación de asimilar transformaciones funcionales y organizativas a partir de los recursos tecnológico-constructivos que constituyen su materialidad. En este sentido, el Seminario-Taller ha retomado para la vivienda privada, los principales instrumentos de la Tesis Doctoral "adaptabilidad y vivienda de producción estatal: estrategias y recursos proyectuales. SubPrograma de Urbanización de Villas y Asentamientos Precarios. 2005-2009"

Considerando estos antecedentes, y a pesar de que la disciplina arquitectónica, en un proceso de actualización permanente continúa explorando nuevas posibilidades constructivas, podemos afirmar que existe una distancia considerable entre las dinámicas en los diferentes modos de habitar (Sarquis,2005) que definen el programa funcional de la vivienda contemporánea, y la implementación de mecanismos que, en la praxis, construyan respuestas alternativas y viables.

\section{MARCO TEÓRICO-METODOLÓGICO}

Respecto del enfoque teórico, sostenemos que la Investigación Proyectual resulta una concepción pertinente para el abordaje de la temática. Este marco conceptual se presenta como una epistemología de y para la arquitectura, anclada en su propia historia y propone pautas metodológicas que reconocen al proyecto como herramienta válida para la producción de conocimientos útiles a los fines disciplinares. Desde aquí comprendemos tectónica y considerando la articulación aspectos que proponen las definiciones de dos referentes vinculados a la producción de conocimiento en arquitectura. El primero, nos ofrece una voz desde el campo de la Historia y señala que el término remite a la visión de la arquitectura como técnica constructiva (Frampton, 1999). El segundo comprende tectónica como "[...] la expresión que la arquitectura realiza con los materiales físicos que elabora" [Sarquis, 2007:56]. Es por esto que podemos definir entonces tectónica como la cualidad estética, sensorial y fundamentalmente, expresiva que un proyecto arquitectónico adquiere cuando pone de manifiesto los recursos constructivos, que mediante los procedimientos específicos -técnicas- constituyen el planteo de su resolución tecnológica como decisión integral. 
Técnica, tectónica y tecnología en el proyecto de la vivienda contemporánea: Una experiencia pedagógica desde la práctica docente de grado

Fiscarelli

A partir de estas nociones el seminario-taller propuso trazar un un renovado y creativo intercambio, con el objeto de ponderar el rol de la tecnología en la definición conceptual de la vivienda contemporánea, y la vez cuestionar críticamente ciertos cánones que aún prevalecen a la hora de materializar la vivienda urbana en la actualidad.

\section{DESARROLLO}

Los contenidos, presentados en una interrelación entre teoría y práctica, se estructuran sobre los siguientes ejes temáticos:

1. El proyecto arquitectónico como herramienta de producción de conocimiento.

¿Qué es investigar en arquitectura? La Investigación Proyectual un marco epistemológico específico. Categorías teóricas centrales. Breves lineamientos metodológicos.

2. El proyecto de la vivienda unifamiliar ante los nuevos desafíos de la arquitectura.

Nuevos modos de habitar y la interdependencia entre cambios durante el Ciclo Vital de las familias en la actualidad y los aspectos físico-espaciales de las viviendas. Oferta y demanda en relación los usuarios y sus dinámicas: vivienda y ámbitos para el trabajo. Las formas alternativas de organización familiar. Las parejas ensambladas y sus demandas funcionales. La creación de ámbitos para lo eventual, lo transitorio como premisa de proyecto. La optimización de la superficie de la vivienda en términos de eficiencia eficiencia topológico-organizativa: estrategias, recursos proyectuales, pautas tecnológicas y criterios dimensionales.

3. Los sub-sistemas constructivos de la vivienda en relación a las dinámicas familiares:

$\begin{array}{ll}\text { - } & \text { El núcleo de servicios } \\ \text { - } & \text { Las particiones } \\ \text { - } & \text { La estructura } \\ \text { - } & \text { El evolvente } \\ \text { - } & \text { El soporte }\end{array}$

Las acciones dinámicas asociadas a los subsistemas: mover, desarmar, ampliar, etc.

La categorización de los recursos proyectuales:

- tecnológico-constructivos,

- morfológico-dimensionales

- topológico-organizativos.

Pautas proyectuales en relación con la edilicia. Coordinación modular y dimensional. Previsión de vanos, sobredimensionamiento estructura, paneles removibles, etc. La posición del núcleo de servicio. Instalaciones complementarias. Eficiencia constructiva.

\section{DINÁMICA DE TRABAJO}

Se organizaron las actividades para 3 encuentros intensivos. La caracterización de las tareas o "bloques" para cada jornada, ha considerado cada una de las variables para la definición de la problemática de abordaje. El objetivo práctico final fue la formulación de una propuesta proyectual para 
Técnica, tectónica y tecnología en el proyecto de la vivienda contemporánea: Una experiencia pedagógica desde la práctica docente de grado

Fiscarelli

una vivienda en lote propio de hasta 100 metros cuadrados. Como técnicas de se trabajaron esquicios, bocetos y maquetas de estudio en materiales livianos.

La dinámica de trabajo, según cronograma de actividades ha sido la siguiente:

- El día 1 habilitó un breve intercambio inicial, con la intención de descubrir las dinámicas cotidianas que redefinen en la actualidad, la concepción del hábitat urbano. ¿Qué demandas específicas sugiere el programa de la vivienda contemporánea? Se propuso un breve repaso de referentes internacionales, latinoamericanos y de la Argentina.

Se trabajó sobre los aspectos particulares de las propuestas: tipologías, subsistemas, resolución constructiva y elementos tectónicos. Se inició la jornada con una exposición teóricas sobre estrategias proyectuales a partir de referentes. Se re- visitaron en perspectivas históricas los conceptos "flexibilidad", "crecimiento" y "versatilidad". Comenzamos con casos instrumentales de Europa y los años '20, asociados con la vivienda mínima en las políticas habitacionales: se discutió el prototipo como vivienda de producción masiva.

Luego se presentó el panorama latinoamericano en torno de los años 60: nuevos y particulares conceptos asociados con instancias espacio-temporales: "etapabilidad", y tipologías de producción social del hábitat vinculadas a sectores postergados respecto del acceso al suelo y a la vivienda: "vivienda semilla", "vivienda galpón", y otras. Breve repaso de los sistemas constructivos de naturaleza abierta o flexible.

Asimismo, se analizó el legado del Proyecto Moderno y las estrategias del espacio flexible. En este sentido, se ponderó el rol de la tecnología en la transformación eventual de la configuración espacial de las viviendas: descripción de los dispositivos técnicos involucrados: planta libre, tabiquería móvil, aspectos topológicos del núcleo de servicios, ámbitos polivalentes, etc.-

- El día 2 propuso una introducción teórica sobre formas alternativas en los grupos familiares, y abrió al debate sobre sus demandas funcionales particulares. Se abordaron las nociones de Vida útil, Ciclo Vital Familiar, Estrategias Familiares de Vida, entre otros conceptos de orden sociológico. El desarrollo discurrió en torno de la tensión ¿Vivienda tipo sin familia tipo? Se analizaron colectivos de usuarios atípicos, y segmentos etáreos convivientes en diversos conjuntos colectivos internacionales.

La jornada estuvo dedicada a los aspectos sociales vinculados con las familias y los modos de habitar en la actualidad. Como parte de las ejercitaciones práctica del bloque de trabajo en taller, se propuso seleccionar de las siguientes situaciones de grupos convivientes, un caso para desarrollar durante el seminario.

1. La situación de padres conviviendo con hijos próximos a migrar de la casa.

2. La situación de trabajar eventualmente en casa, con tares que necesitan de un ámbito similar a una oficina o taller.

3. La situación habitacional de un colectivo intergeneracional de personas que comparten la vivienda, pero que alternativamente demandan mayor o menor cantidad de espacio habitable por ciclos temporales.

4. La situación de uno de los miembros de una pareja recientemente divorciada, que recibe eventualmente a los hijos del matrimonio.

- La situación de la pareja que ha resuelto disolver su unión, pero que aún debe compartir la vivienda. Luego los estudiantes abordaron la definición ficcional10 de un programa de actividades para el grupo de usuarios con el objeto de prever las futuras posibles modificaciones en la vivienda a desarrollar. El debate al interior de los grupos de trabajos giró 
Técnica, tectónica y tecnología en el proyecto de la vivienda contemporánea: Una experiencia pedagógica desde la práctica docente de grado

Fiscarelli

en torno del interrogante ¿Cómo se presentan los nuevos encargos funcionales según el habitar actual?

- El día 3 estuvo dedicado en particular a definir los aspectos materiales de la vivienda. Comenzamos con una exposición teórica: el rol de la materialidad en la definición de una tectónica para la vivienda contemporánea.

Se repasaron experiencias alternativas que contribuyeron a la (re) definición de la vivienda contemporánea desde la tecnología. Se dedicó el bloque de trabajo taller a la exploración material, conceptual y tectónica de las propuestas a desarrollarse.

Al final de la jornada se produjo una ronda de intercambios, exposición y síntesis. El cierre, a cargo de los docentes involucrados habilitó el debate y reflexiones sobre los productos. Se planteó un ejercicio de auto-evaluación grupal.

\section{DISCUSIÓN DE LOS RESULTADOS | PRESENTACIÓN DE LOS CASOS REFERENCIALES}

Como resultado de las indagaciones, proponemos interpelar los procesos proyectuales en torno de los conceptos técnica, tecnología y tectónica. En este sentido, recuperamos casos instrumentales que permitirán ilustrar el alcance de las propuestas, asociándolos con temáticas de investigación específicas del programa contemporáneo de la vivienda. A saber:

- La vivienda productiva. La familia que despliega actividades laborales en el hogar.

- La vivienda "nido vacío". La adecuación funcional frente al eventual "decrecimiento".

- La vivienda "régimen de visitas". El uso transitorio de ámbitos para visitas de hijos.

Vale recordar que los planteos funcionales remite a configuraciones "ficcionales" determinadas por grupos convivientes problematizados.

a. La vivienda productiva [Figura 1]

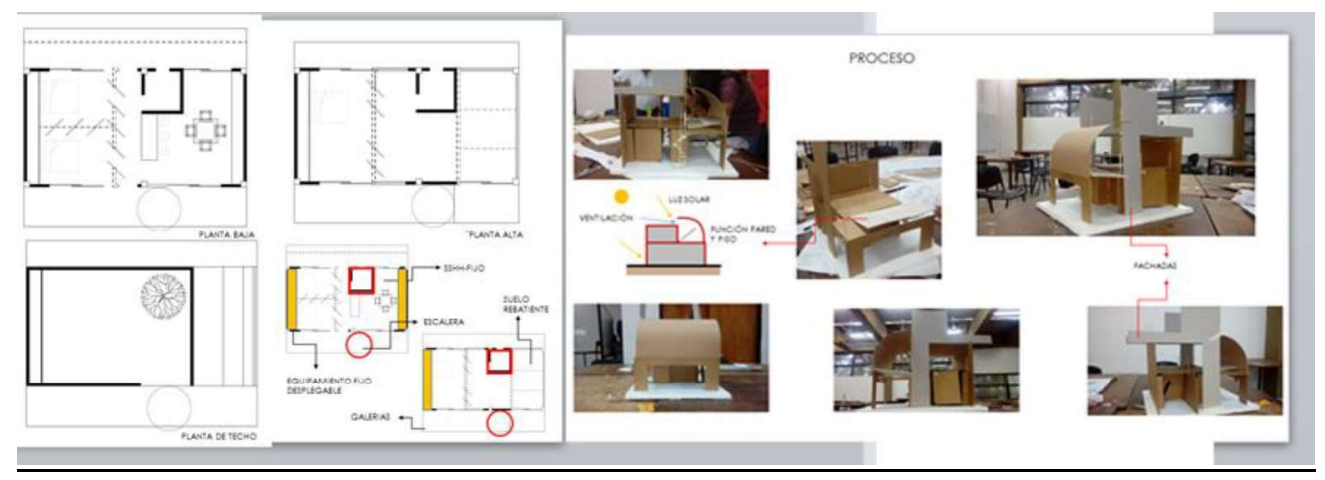

Figura 1. Equipo 9. "La vivienda productiva".

Para la situación de personas que necesitan un ámbito para trabajar eventualmente en casa, el grupo 9 partió de la modalidad "vivienda semilla", analizando sus componentes organizativos como estructura crecedera. Reconocieron además que los ingresos que surgieran de las actividades desarolladas en el ámbito de trabajo servirían para materializar la etapa final de la vivienda. Determinaron que se trataría de la vivienda de uno de los miembros de una pareja divorciada que, según un régimen de visitas, recibiría a dos hijos adolescentes. 
Técnica, tectónica y tecnología en el proyecto de la vivienda contemporánea: Una experiencia pedagógica desde la práctica docente de grado

Fiscarelli

\section{Estrategias:}

1. Consolidar la posición del núcleo sobre el soporte en relación a dos "bandas" opuestas relacionadas con los apoyos o equipamiento. A partir de estos elementos, establecer una grilla de base modular.

2. Distribuir los ámbitos modulares sobre la planta baja en forma polivalente, habilitando la posibilidad de intercambiar sus usos -estar, área de trabajo, estudio, taller.

3. Considerar una eventual doble altura que se "conquista gracias a un entrepiso móvil. La envolvente toma la forma del recorrido del entrepiso, a modo idea tectónica con rasgos maquínicos o funcionalistas.

4. Diferenciar como toma de partido las áreas de "espacio permanente" de las que remiten a modalidades "polivalentes" o de múltiples usos.

Respecto de la tectónica, los estudiantes del equipo plantearon una volumetría transparente que anuncia los movimientos dinámicOs del interior. Han sugerido que parte de la premisa de proyecto resulta de visibilizar los tiempos acelerados de la vida cotidiana, y por consiguiente los subsistemas particiones y envolvente han resultado protagonistas en su articulación fundamental. Interesante planteo gráfico que ofrece el grupo en tanto propone fusión entre "pared" y "piso", como recurso proyectual tectónico que materializa la idea de recorrido interior según instancias temporales.

\section{b. La vivienda "nido vacío" [Figura 2]}

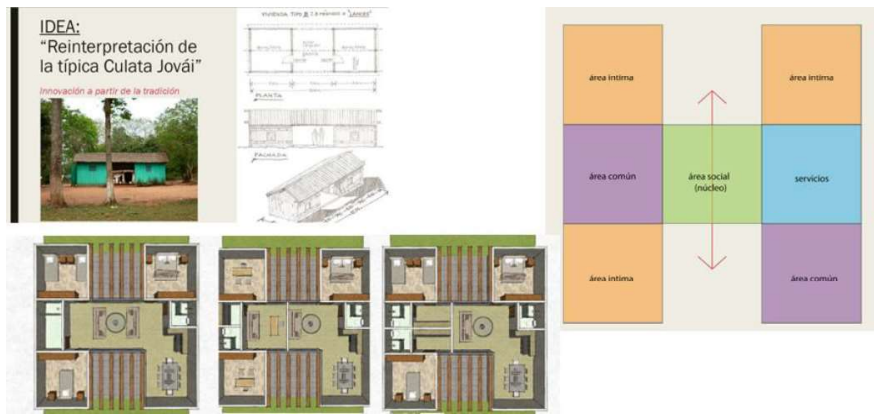

Figura 2: Equipo 12. "La vivienda nido vacío"

Interesante propuesta por partida doble: por un lado, propone resoluciones para adecuar los ámbitos originales de la vivienda luego de que los hijos adultos migren definitivamente del hogar familiar. Al mismo tiempo, recupera una tradición originaria de hábitat guaraní.

El equipo nos habla de "innovación a partir de la tradición" y consolida una reintepretación de la "culata jovài”, configuración espacial autóctona de las comunidades originarias habitantes del Paraguay.

Como estrategias plantean:

Recuperar la disposición tradicional que propone un área central común y a cada extremo los ámbitos íntimos.

a. Consolidar el centro atravesable como un núcleo de reunión familiar.

b. Establecer una modulación del soporte que determina equivalencias en los ámbitos: con esto se propicia una polivalencia, es decir que se sugiere la posibilidad de eventualmente intercambiar los usos -un dormitorio pasaría a ser una salita de estar, por ejempolo.

c. Se ubican en posición estratégica los servicios, como un componente lateralizado y equidistante respecto de las áreas íntimas.

d. Se configura una nueva "galeria" como un margen dimensional susceptible de convertirse en un nuevo espacio de uso interior. ¿Qué pasa si alguno de los hijos vuelve con su pareja? 
Técnica, tectónica y tecnología en el proyecto de la vivienda contemporánea: Una experiencia pedagógica desde la práctica docente de grado

Fiscarelli

Nos encontramos frente a un caso que discute lo organizativo de la vivienda en torno de la nueva situación problemática pero que rescata los aspectos tecnológicos tradicionales: estructura, modulación, muros portantes y prácticamente la ausencia de divisorias. Resulta un planteo funcional esquemático pero eficiente, que pondera la existencia de "espacios intermedios" incluso cuando propone una compacidad intencional. Han registrado como parte de la memoria técnica la "etapabilidad" como premisa de proyecto, pero atendiendo al grupo conviviente, han capitalizado la reversabilidad como estrategia de diseño.

Asimismo, los autores, retomando las fortalezas de la tipología originaria, rescatan los beneficios de la configuración en términos de eficiencia energética: señalan la ventilación cruzada como un valor esencial.

\section{c. La vivienda "régimen de visitas" [Figura 3]}

El equipo ha trabajado sobre la situación habitacional de un padre que recibe eventualmente los hijos, bajo un régimen de visitas. ¿Qué sucede con los espacios adicionales cuando no están ocupados?

Como estrategias principales han mencionado:

a. Reconocer conceptualmente un programa mínimo a partir del usuario que determina el programa.

b. Considerar la "galería" como ámbito de expansión por excelencia, es decir como un margen dimensionar que se puede ocupar o convertir en interior gracias a un cerramiento móvil.

c. Determinar estratégicamente la posición de la estructura portante.

d. Replicar el planteo de planta alta en el nivel superior, con la intención de habilitar un uso polivalente de los espacios en sección.

e. Diseñar un modelo de evolvente "fuelle", incorporando dispositivos técnicos al tradicional muro de ladrillos.

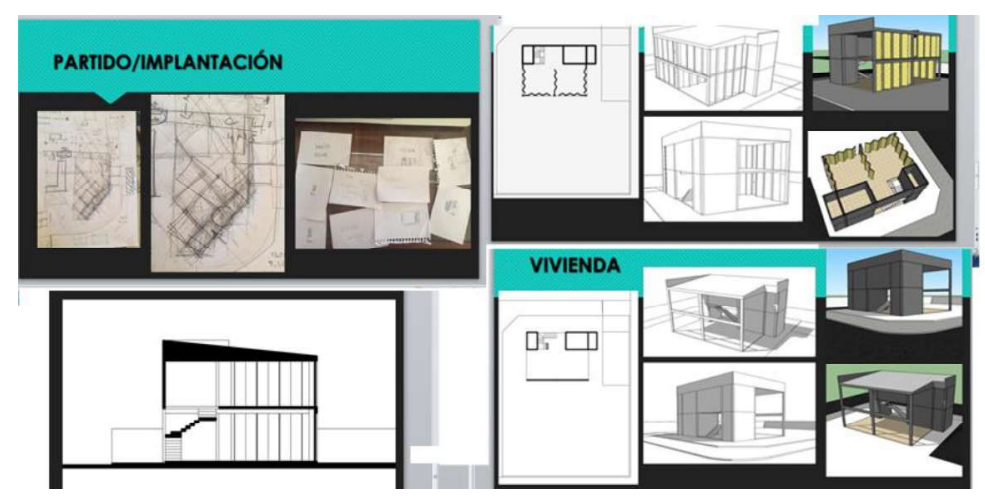

Figura 3. Equipo 14. "La vivienda régimen de visitas"

Han formulado un práctico modelo conceptual, que recupera las nociones principales del alojamiento mínimo, concentrado en torno de una minuciosa compacidad. Esta decisión obligó al grupo a formular un estudio dimensional de los elementos que topológicamente se disponen sobre el soporte.

Resulta particularmente interesante el diseño de los paneles de cerramiento de la envolvente, que constituyen la solución técnica que determina el carácter expresivo de la propuesta. Es decir, el carácter tectónico del modelo asociado con la movilidad temporal que propone el crecimiento-decrecimiento. 


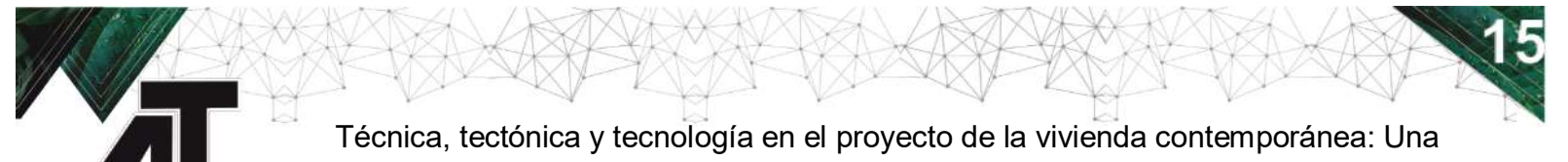
experiencia pedagógica desde la práctica docente de grado

Fiscarelli

\section{CONCLUSIONES}

La dinámica del taller instaló la terminología específica para comenzar asociar criterios funcionales con resoluciones constructivas. Como resultado han sido notables las reinterpretaciones de casos del contexto latinoamericano que han sido interpelados respecto de las posibilidades que ofrecen en relación con las demandas del habitar contemporáneo.

En segundo lugar, la estructura que ha constituido la propuesta pedagógica permitió interpelar las prácticas proyectuales de los equipos según un criterio de complejidad creciente: tipologías, modelos conceptuales y estrategias, luego usuarios y dinámicas familiares y por último, resoluciones matéricas y síntesis. Esto no ha sido en detrimento de una concepción integral de los planteos proyectuales, sino como instancias de aproximación secuencial al abordaje de la problemática que plantea el ejercicio. Como consecuencia del proceso, ha sido sumamente enriquecedor el constante debate que habilitaron los bloques prácticos en torno de las diversas alternativas que, en la actualidad, construyen nuestras realidades sociales latinoamericanas. No nos referimos sólo a la configuración del hábitat urbano, sino a la diversidad de agrupaciones familiares que aún no han sido visibilizadas ni aprehendidas por las prácticas proyectuales de la formación académica.

Por último, señalar que la tectónica en su acepción framtoniana, es decir como expresión de la lógica constructiva, ha provocado el despliegue de una interesante gama de soluciones que, fundamentalmente socavan el carácter permanente de la arquitectura. En otros términos, los imaginarios [Londoño en Sarquis, 2007] anclados en la cultura técnica de la comunidad se vieron confrontados con el carácter eventual de las pautas funcionales. El ladrillo -y consecuentemente, la construcción tradicional- se vio enfrentado con dispositivos dinámicos que garantizaban la flexibilidad. En este sentido, tanto el detalle constructivo como la exploración volumétrica han ocupado un lugar central en el desarrollo de las propuestas.

En lo específico, y en relación con las nociones técnica, tecnología y tectónica, las propuestas han sabido explorar el rol de los recursos constructivos en la configuración de propuestas adaptables. Hemos valorado la inevitable asociación entre materiales, procedimientos, lenguaje expresivo y estrategias proyectuales atravesadas por dinámicas temporales particulares. Los tres conceptos estuvieron articulados fundamentalmente por las ideas de "flexibilidad", "crecimiento" y "versatilidad" o "polivalencia" funcional de los ámbitos. Esta intencional fusión se ha constituido en premisa para la definición de los aspectos cualitativos de las propuestas, en términos de adaptabilidad. [Fiscarelli, 2016]

Como síntesis, podemos afirmar que el proceso de desarrollo del workshop ha contribuido fundamentalmente a interpelar sus propias prácticas proyectuales, retomando la necesaria articulación diseño-tecnología, y consecuentemente se ha promovido un espacio para la construcción de herramientas disciplinares específicas para abordar el programa funcional de la vivienda contemporánea. 
Técnica, tectónica y tecnología en el proyecto de la vivienda contemporánea: Una experiencia pedagógica desde la práctica docente de grado

ARQUITECND

Fiscarelli

\section{BIBLIOGRAFÍA}

Bertuzzi, D. (2007) "Adaptabilidad es más". Mar del Plata. FUDEM.

Sarquis, J. (2005) "Arquitectura y Modos de Habitar." Buenos Aires. Ed. Nobuko

Torrado, S. (2003) "Historia de la familia argentina moderna (1870-2000)". Buenos Aires. De la Flor.

Fisch, S.; Pagani, G.; Etulain, J. (2014) "Vivienda Contemporánea: estrategias de proyecto". La Plata. Ed. Edulp

Frampton, K. (1999) "Estudios sobre la cultura tectónica. Poéticas de la construcción en la arquitectura de los Siglos XIX y XX." Madrid. Ed. Akal.

Sarquis, J. (2007) "Itinerarios del proyecto. La Investigación Proyectual como forma de conocimiento en arquitectura". Buenos Aires. Ed. Nobuko. 